\title{
Repeatable differences in exploratory behaviour predict tick infestation probability in wild great tits
}

\author{
Robert E. Rollins ${ }^{1}$ (D) Alexia Mouchet ${ }^{2} \cdot$ Gabriele Margos $^{3} \cdot$ Lidia Chitimia-Dobler $^{4} \cdot$ Volker Fingerle $^{3} \cdot$ \\ Noémie S. Becker ${ }^{1} \cdot$ Niels J. Dingemanse ${ }^{2}$
}

Received: 22 June 2020 / Revised: 3 January 2021 / Accepted: 5 January 2021 / Published online: 10 February 2021

(C) The Author(s) 2021

\begin{abstract}
Ecological factors and individual-specific traits affect parasite infestation in wild animals. Ixodid ticks are important ectoparasites of various vertebrate hosts, which include passerine bird species such as the great tit (Parus major). We studied various key ecological variables (breeding density, human disturbance) and phenotypic traits (exploratory behaviour, body condition) proposed to predict tick infestation probability and burden in great tits. Our study spanned 3 years and 12 nest box plots located in southern Germany. Breeding, adult great tits were assessed for exploration behaviour, body condition, and tick burden. Plots were open to human recreation; human disturbance was quantified in each plot as a recreation pressure index from biweekly nest box inspections. Infested individuals were repeatable in tick burden across years. These repeatable among-individual differences in tick burden were not attributable to exploration behaviour. However, faster explorers did have a higher infestation probability. Furthermore, body condition was negatively correlated to tick burden. Recreation pressure was correlated to increased infestation probability, although this relationship was just above the threshold of statistical significance. Our study implies that avian infestation probability and tick burden are each determined by distinct phenotypic traits and ecological factors. Our findings highlight the importance of animal behaviour and human disturbance in understanding variation in tick burden among avian hosts.
\end{abstract}

\section{Significance statement}

Various abiotic and biotic factors, including personality type, influence tick parasitism in birds, but exactly how all these factors interplay remains unclear. We studied a wild population of great tits over a 3-year period and assessed birds for their exploration behaviour and tick infestation. We found that more explorative great tits were more likely to be infested with ticks. By contrast, faster explorers did not have higher tick burdens. Tick burden was nevertheless moderately repeatable among individuals. Our results imply that animal personality influences the probability of parasite infestation, and that infestation likelihood versus intensity are determined by distinct mechanisms. Our work highlights the importance of animal behaviour to understand parasite infestation in wild populations.

Keywords Animal personality · Parasite-host interactions · Host-tick relationships · Infestation probability · Tick burden . Urbanisation

Communicated by K. van Oers

Robert E. Rollins

rollins@bio.lmu.de

1 Division of Evolutionary Biology, Faculty of Biology, LMU Munich, Grosshaderner Str. 2, 82152 Planegg-Martinsried, Germany

2 Behavioural Ecology Group, Ludwig-Maximilians University Munich/Department Biology II, Grosshaderner Str. 2, 82152 Planegg-Martinsried, Germany

3 National Reference Center for Borrelia, Bayerisches Landesamt fuer Gesundheit und Lebensmittelsicherheit (LGL), Oberschleissheim Office, Veterinaerstr. 2, 85764 Oberschleissheim, Germany

4 Bundeswehr Institute of Microbiology, Munich, Germany

\section{Introduction}

Parasitic relationships between obligate ectoparasites and their animal hosts are predicted to be influenced by ecological factors and host-specific traits (Barber and Dingemanse 2010; Kiffner et al. 2011; Perez et al. 2016). Ticks are obligate ectoparasites that feed on the blood of various vertebrate hosts, including birds (Hillyard 1996; Kurtenbach et al. 2006), and negatively influence host fitness (Norte et al. 2013). Tick burden varies among individuals as a function of individualspecific traits, such as host foraging style, personality 
(Barber and Dingemanse 2010; Newman et al. 2015; Sih et al. 2018), body size, or body condition (Norte et al. 2013; Newman et al. 2015). For instance, ground-foraging (compared to canopy-foraging) bird species have higher tick burdens (Newman et al. 2015; Loss et al. 2016; Kocianová et al. 2017), as do larger (compared to smaller) species (Newman et al. 2015). Furthermore, individual-specific differences in foraging or social behaviour can also predict tick burdens within populations of the same species (Sih et al. 2018). For example, individuals that are more active and explorative in their (foraging) behaviour may encounter more parasites (e.g. ticks), and consequently have higher tick burdens (Barber and Dingemanse 2010; Sih et al. 2018; Taggart et al. 2018). The link between exploration behaviour and ectoparasite (i.e. tick) infestation has been investigated in few species (Barber and Dingemanse 2010; Sih et al. 2018); further studies are needed to determine whether reported relationships are general versus specific to species or their ecological conditions. Here, we investigated whether tick infestation varied as a function of two individual-specific traits (exploratory behaviour and body condition), using wild great tits (Parus major) as a model. Both exploration behaviour and body condition are repeatable in great tits (Stuber et al. 2013; Moiron et al. 2018, 2019).

The likelihood and number of ticks carried by an individual bird can vary also as a function of ecological factors, particularly those affecting tick abundance and questing behaviour (Mejlon and Jaenson 1997). Key factors include temperature and humidity (Lindgren et al. 2000; Kiffner et al. 2011; Titcomb et al. 2017), host breeding density (Oorebeek and Kleindorfer 2008; Takumi et al. 2019), and vegetation type and coverage (Kiffner et al. 2011; Tack et al. 2012a, b, 2013). These factors vary spatiotemporally and, consequently, tick abundance will vary throughout the environment and affect host tick burden depending on how hosts are distributed over tick habitats. Tick burden generally varies with tick and host density (Randolph 1998, 2008); any factor that affects their ratio thus influences tick burden. Both the percentage of infested birds and tick burden are, consequently, predicted to change with habitat modifications and climate change (Lindgren et al. 2000; Randolph 2004; Akimov and Nebogatkin 2016). For example, city-dwelling birds may carry lower tick burdens (Evans et al. 2009; Hamer et al. 2012) as urbanisation reduces tick densities (Akimov and Nebogatkin 2016; Heylen et al. 2019). To date, most research has focused on the effects of urbanisation on bird tick infestation due to habitat modifications rather than susceptibility to tick infestation (Hamer et al. 2012; Akimov and Nebogatkin 2016). Human presence affects bird reproductive success and bird habitat choice (Hutfluss and Dingemanse 2019), and may therefore influence susceptibility of birds to tick infestation. Studies documenting effects of human disturbance on tick burden in bird populations are required to identify factors with general versus study-specific effects (Kelly 2006; Nakagawa and Parker 2015).

We tested the link between two phenotypic traits (exploration behaviour and body condition) and two environmental factors (breeding density and human disturbance) on tick infestation of great tits inhabiting 12 forest plots varying in breeding density and human disturbance levels (Araya-Ajoy and Dingemanse 2017; Hutfluss and Dingemanse 2019). Our repeated measures design spans over 3 years and 12 plots and enables the quantification of the amount of variance in tick burden attributable to among-year (temporal variation), among-plot (spatial variation), and among-bird (individual variation) variation. This variance partitioning approach thereby resulted in quantitative estimates of the magnitude of variation attributable to multiple key biological levels of variation.

\section{Material and methods}

\section{Study sites and data collection}

Our study was performed in 12 nest box plots within a $10 \times 15$ $\mathrm{km}^{2}$ area in southern Munich $\left(47^{\circ} 57^{\prime} \mathrm{N}, 11^{\circ} 21^{\prime} \mathrm{E}\right)$ established in autumn 2009. Each plot was fitted with 50 nest boxes in a regular grid covering approximately 9 hectares. Breeding parameters of great tits (detailed below) were monitored for 3 years (2017-2019) within all plots except one plot that was monitored only in 2017. Each nest box was inspected biweekly during the breeding season (April-July) for nesting activity. During plot inspections, the number of recreationists was counted as a measure of human disturbance (detailed by Hutfluss and Dingemanse 2019).

When hatchlings were 10-12 days old, both parents were captured in the nest box using a spring trap. Birds were given a unique, numbered band if not previously banded. They were then tested for their exploration behaviour (detailed below), weighed, measured morphologically (Moiron et al. 2019), aged (based on plumage characteristic as first year breeder or older; Dingemanse et al. 2020), and screened for ticks (protocol detailed below). Most birds were captured in May and a few in June. These captures were used to calculate breeding density, defined as the number of breeding pairs producing first clutches per hectare.

\section{Exploration behaviour}

Exploration behaviour was measured using a novel environment test adapted to the field using established protocols (see Stuber et al. 2013). Birds were transferred to a holding box attached to a cage $(61 \mathrm{~L} \times 39 \mathrm{~W} \times 40 \mathrm{H} \mathrm{cm})$ fitted with a mesh 
front and three perches, representing the novel environment. The holding box was covered with a cloth bag and the subject was allowed to acclimatise for $1 \mathrm{~min}$. After acclimatisation, the focal bird was released into the novel environment without handling and recorded for 2 min with the field observer located out of sight. Videos were subsequently scored by dividing the cage into six equal sections and three floor sections as described in Stuber et al. (2013). The exploration score was calculated as the sum of movements between all sections within the first 2 min of entering the novel environment (AbbeyLee and Dingemanse 2019; Dingemanse et al. 2020). The behavioural data used in this paper was based on videos scored in randomised order within years, and blindly with respect to individual identity and tick infestation.

\section{Tick burden and collection}

Ticks tend to concentrate around the eyes and beak of great tits (Heylen and Matthysen 2008; Fracasso et al. 2019). A regional patch examination protocol (reviewed in Lydecker et al. 2019) was developed to standardise the screening. We screened (1) around the eyes/ears and along the margin of the beak on both sides of the bird, (2) underneath the beak, (3) along the top margin of the beak, and (4) on the top of the head. We subsequently calculated the total number of ticks carried by each captured bird. In 2018-2019, all captured birds were screened; in 2017 only a sample. In 2018 and 2019, a sample of ticks were collected using fine tweezers and stored in $99 \%$ ethanol as part of another study. Those were subsequently morphologically identified to life-stage and species according to published taxonomic keys (Filippova 1977; Hillyard 1996; Estrada-Peña et al. 2014).

\section{Recreation pressure index}

During each plot inspection, all observers recorded each recreationist seen and their specific location (detailed by Hutfluss and Dingemanse 2019). We took care to count each recreationist only once by only counting the first observation of each recreationist per plot check. The probability to observe a recreationist during a plot inspection is biased by various factors (Hutfluss and Dingemanse 2019). To obtain an unbiased index of recreation pressure, the binary probability to observe recreationists was calculated using all inspections conducted from 2010 to 2019 ( $n=$ 3724 inspections). This probability was calculated using a binomial generalised linear mixed effects model (GLMM) fitting fixed effects for plot inspection duration, the number of observers, and starting time (in hours from sunrise) (Hutfluss and Dingemanse 2019). We fitted random intercepts for each unique combination of plot and year (termed plot-year, see Abbey-Lee et al. 2016; Araya-Ajoy et al. 2016; Araya-Ajoy and Dingemanse 2017) to acquire an average value of recreation pressure for each plot in each year, and for date of observation to control for date-specific environmental effects (Supplemental Table 1). We extracted best linear unbiased predictors (BLUPs) for each plot-year between 2017 and $2019(n=34)$ and used them in subsequent models as a recreation pressure index. The usage of BLUPs has been criticised when associated uncertainty is not taken forward; some have proposed to estimate a posterior distribution of possible BLUP values and thereby take forward uncertainty in subsequent analyses (Hadfield et al. 2010; Houslay and Wilson 2017). However, taking forward uncertainty in BLUP values can result in biased estimates, whereas utilising average BLUPs as fixed effects results in less precise but unbiased estimates (Dingemanse et al. 2020). Therefore, we here present the estimated effect of the average BLUP values.

\section{Data preparation}

The scaled mass index (Peig and Green 2009) was used as our measure of body condition. Following Peig and Green (2009), this index was calculated separately for each sex as follows:

$$
S M_{i}=M_{i} \times\left(\frac{L_{0}}{L_{i}}\right)^{b_{\mathrm{SMA}}}
$$

where $M_{i}$ and $L_{i}$ are the mass and tarsus measurements of individual $i$ respectively; $L_{0}$ is the arithmetic mean of all tarsus lengths of either all females or all males (dependent on the sex of individual $i$ ); and $b_{\mathrm{SMA}}$ is the scaling exponent calculated as:

$$
b_{\mathrm{SMA}}=\frac{b_{\mathrm{OLS}}}{R}
$$

where $b_{\mathrm{OLS}}$ is the slope of linear regression of logtransformed body mass as a function of log-transformed tarsus length of either all females or all males (dependent on the sex of individual $i$ ) and $R$ is the Pearson correlation coefficient of the regression. We used all birds recorded from 2010 to 2019 ( $n=4273$ records) to calculate the scaling coefficient (including any repeated measures).

We calculated individual-mean exploration scores using a linear mixed effects model (LMM) fitting exploration behaviour as the response variable (Supplemental Table 2). We fitted test sequence as a fixed effect covariate to control for sequence effects (Dingemanse et al. 2012, 2020), and included random intercepts for individual and plot-year. The model included data from all years $(2010-2019)(n=4251)$ and assumed a Gaussian error distribution. We extracted an average BLUP value per individual, which we used in subsequent analyses as a measure of an individual's average (experiencecorrected) exploration score. 


\section{Statistical analysis}

Tick infestation of great tits was analysed in two parts using GLMMs. First, infestation probability was estimated using all recorded captures from 2017 through 2019 ( $n=784$ records). Second, tick burden (i.e. the absolute number of ticks observed) was modelled using only infested individuals $(n=$ 520 records). For both response variables, the model set up was the same. Fixed effects were fitted for recreation pressure, breeding density, body condition, sex, age, and individualmean exploration score. Sex and age were fitted because males (versus females) and younger (versus older) birds often have higher tick burdens (Heylen and Matthysen 2008; Heylen et al. 2013). Random intercepts were fitted for year, plot, plot-year, field observer, nest box, and individual. Prior to analysis, all fixed effects were mean-centred, and variance standardised, such that the statistical intercepts of our models reflected the value for the average individual in the average environmental condition.

All statistical analyses were performed in R (version 3.5.3) ( $\mathrm{R}$ Core Team 2019). Models were run using the functions lmer and glmer from the package lme4 (Bates et al. 2015) assuming either binomial (infestation probability) or Poisson (tick burden) error distributions. Mean estimates and their 95\% credible intervals (CIs) were estimated based on 5000 simulations using the sim function from the arm package (Gelman and Su 2016). Residual errors were calculated according to Nakagawa and Schielzeth (2010). Adjusted repeatability values were calculated as the proportion of variance unexplained by the fixed effects that was explained by the focal random effect. Estimates with 95\% CIs not overlapping zero were treated as statistically significant in the frequentist's sense. Models were checked graphically for fit (Supplemental Figs. 1 and 2).

\section{Results}

On average, $65.1 \%$ of all captured adult great tits carried ticks. Infested birds were found in all study plots and all years (Fig. $1)$; the average tick burden of an infested bird $(n=520)$ was (mean $\pm \mathrm{SD}) 5.69 \pm 9.02$ ticks (Fig. 2). Collected ticks $(n=$ $1583)$ were either larvae $(n=860)$ or nymphs $(n=722)$; we found only one (male) adult tick (in 2018). All ticks belonged to the genus Ixodes; the most common species were I. ricinus (43.9\%), I. frontalis $(8.3 \%)$, and I. inopinatus $(2.8 \%)$. The remaining ticks $(45.0 \%)$ could only be identified to genus (Ixodes spp.) and not species level. Infestation probability but not tick burden varied between plots (Table 1). Both tick burden and infestation probability varied among nest boxes (Table 1). Individuals were not repeatable in infestation probability (Table 1). Analyses based on infested individuals showed that tick burden was moderately repeatable (estimate: 0.45; 95\% CI: $0.40,0.51$ ).

Exploration behaviour positively correlated with infestation probability (estimate: 0.18 ; 95\% CI: $0.01,0.35$; Fig. 3) but not with tick burden (estimate: $-0.02 ; 95 \% \mathrm{CI}:-0.12$, 0.09 ). Additionally, body condition was negatively correlated with tick burden (estimate: $-0.11 ; 95 \%$ CI: $-0.19,-0.02$; Fig. 4) but not with infestation probability (estimate: 0.06 ; 95\% CI: $-0.11,0.24)$. A positive trend between infestation probability and recreational pressure was observed but was not statistically significant (estimate: $0.21 ; 95 \% \mathrm{CI}:-0.03$, 0.43 ; Fig. 5). Breeding density, age, and sex did not affect infestation probability or tick burden (Table 1).

\section{Discussion}

The relationship between ticks and their avian hosts is shaped by ecological factors that influence both host population dynamics and parasite (tick) distributions. Their respective

Table 1 Effect size estimates ( $\beta$ ) and 95\% credible intervals (CIs) for great tit tick infestation as the probability of infestation ( $n=784$ records) and tick burden of infested individuals ( $n=520$ records)

\begin{tabular}{lll}
\hline & Infestation probability & Tick burden \\
\hline Fixed effects & $\beta(95 \% \mathrm{CI})$ & $\beta(95 \% \mathrm{CI})$ \\
Intercept & $0.89(0.42,1.37)$ & $1.20(0.75,1.64)$ \\
Recreational pressure & $0.21(-0.03,0.43)$ & $-0.07(-0.22,0.08)$ \\
Breeding density & $0.08(-0.26,0.43)$ & $-0.04(-0.25,0.17)$ \\
Exploratory behaviour & $0.18(0.01,0.35)$ & $-0.02(-0.12,0.09)$ \\
Body condition & $0.06(-0.11,0.24)$ & $-0.11(-0.19,-0.02)$ \\
Sex & $-0.06(-0.42,0.24)$ & $-0.06(-0.24,0.12)$ \\
Age & $-0.07(-0.24,0.09)$ & $0.01(-0.08,0.10)$ \\
Random effects & $\sigma^{2}(95 \% \mathrm{CI})$ & $\sigma^{2}(95 \% \mathrm{CI})$ \\
Year & $0.04(0.00,0.17)$ & $0.13(0.03,0.36)$ \\
Plot & $0.47(0.18,0.94)$ & $0.05(0.02,0.09)$ \\
Plot-year & $0.08(0.04,0.12)$ & $0.07(0.04,0.12)$ \\
Observer & $0.01(0.00,0.01)$ & $0.13(0.06,0.23)$ \\
Nest box & $0.64(0.55,0.74)$ & $0.28(0.23,0.32)$ \\
Individual & $0.00(0.00,0.00)$ & $0.45(0.40,0.51)$ \\
Residual & $3.45(3.45,3.36)$ & $1.27(1.39,1.18)$ \\
Adjusted repeatability & $r(95 \% \mathrm{CI})$ & $r(95 \% \mathrm{CI})$ \\
Year & $0.01(0.00,0.03)$ & $0.05(0.01,0.13)$ \\
Plot & $0.10(0.04,0.18)$ & $0.02(0.01,0.03)$ \\
Plot-year & $0.02(0.01,0.02)$ & $0.03(0.02,0.04)$ \\
Observer & $0.00(0.00,0.00)$ & $0.05(0.03,0.08)$ \\
Nest box & $0.14(0.13,0.14)$ & $0.12(0.11,0.11)$ \\
Individual & $0.00(0.00,0.00)$ & $0.19(0.18,0.18)$ \\
Residual & $0.74(0.82,0.63)$ & $0.53(0.64,0.42)$ \\
\hline a & & \\
\hline & &
\end{tabular}

${ }^{\mathrm{a}}$ Differences between sexes (reference category $=$ female) 
effects, furthermore, vary as a function of phenotypic traits expressed by hosts, such as their behavioural (or 'personality') types. Our study sheds light on the relative influence of each of these biological factors. We quantified effects of human disturbance, breeding density, and phenotypic traits specific to hosts (exploration behaviour and body condition), using a cavity-breeding passerine, the great tit, as a model. We found that faster explorers were more likely to be infested with ticks. Furthermore, infested birds had higher tick burdens when in poor condition. Additionally, we found a non-significant trend suggesting that high recreation pressure increased infestation probability. Finally, both tick burden and infestation probability varied across time (years) and space (plots). Importantly, among infested birds, tick burden was moderately repeatable across years, implying that individual phenotypic traits (other than exploration behaviour and body condition) influenced tick infestation. Our findings help elucidate the importance of ecological and behavioural mediators of the relationship between ticks and their avian hosts. This observational study now awaits experimental testing to reveal causality of the reported relationships.

Explorative birds were more likely to be infested but did not carry more ticks. This very same pattern has also been reported in wild squirrels (Santicchia et al. 2019). Our birds were predominantly infested by larvae and nymphs of the genus Ixodes. As for many other vertebrate hosts in Europe (Hillyard 1996), the most common tick species was I. ricinus. This tick species quests for hosts vertically up to $1.5 \mathrm{~m}$ (Mejlon and Jaenson 1997). An explanation for our results that requires verification is that explorative birds thus foraged close to the ground, where nymphs and larvae are most abundant (Mejlon and Jaenson 1997), and therefore were more likely to acquire ticks. Indeed, foraging style has previously been shown to be an important predictor of tick burden in other bird species (Newman et al. 2015; Kocianová et al. 2017). Exploration behaviour is known to predict foraging style, and therefore, the observed effect of exploration behaviour could partially be a result of individual differences in foraging style (Verbeek et al. 1994; Aplin et al. 2014; Moiron et al. 2018; but see Moyers et al. 2018). The use of radio-tracking may help to elucidate whether increased infestation probability is linked to where faster explorers forage or to how much they move (Snijders et al. 2014).

Sex and age did not influence tick infestation even though previous studies found (or implied) that males (compared to females) have increased tick burdens (Hoodless et al. 2003), while older (versus younger) birds have decreased tick burdens (Heylen et al. 2013; but see Heylen and Matthysen 2008). The absence of a sex effect in this study could relate to our study design. We collected data during the breeding season, when both males and females may have been in low condition due to chick rearing (Ots and Horak 1996), which may have masked any sex differences. Even so, sex-biased parasite infestation is not universally found in all species, suggesting that our null effect is genuine (Heylen and Matthysen 2008; Kiffner et al. 2013). Additionally, we found no effect of age on tick infestation in agreement with another study on great tits (Heylen and Matthysen 2008; but see Heylen et al. 2013). The lack of an effect of bird age could stem from within- versus among-individual age effects having opposite signs (van de Pol and Wright 2009). To test if this was the case, we split age into among- and within-individual components (Supplemental Text 1). This level-specific analysis also failed to show effects of these factors (Supplemental Text 1) supporting the absence of an age effect in our data.

Other research has argued that tick burden positively correlates to body condition (Mysterud et al. 2015). However, correlations between parasite burden and body condition occur in some but not all species, and are thus most likely not universal (Kiffner et al. 2013). How body condition of great tits relates to their tick infestation remains to be determined (Heylen and Matthysen 2008; Heylen et al. 2013).
Fig. 1 Percentages of all captured great tit adults infested with ticks from the 2017 to 2019 breeding seasons. Data for plot 7 were only available for the year 2017

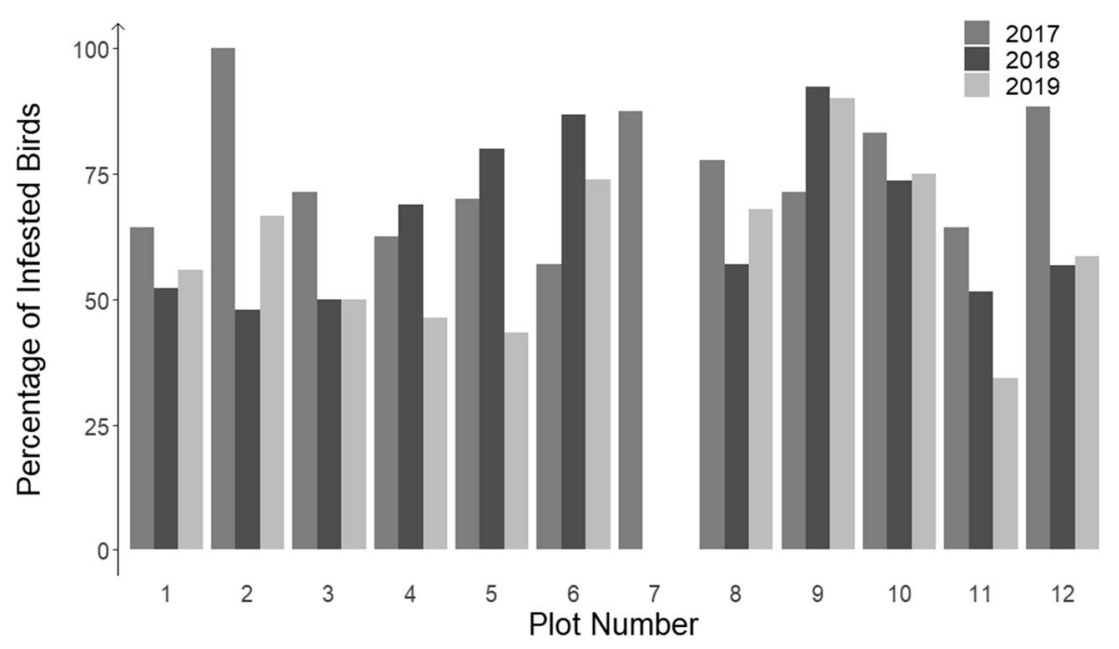


Fig. 2 Average tick burden per infested great tit adult captured in each study plot during each field season (years 2017-2019). Error bars represent the standard error. Numbers above the bars are the number of great tits captured. Data for plot 7 were only available for the year 2017

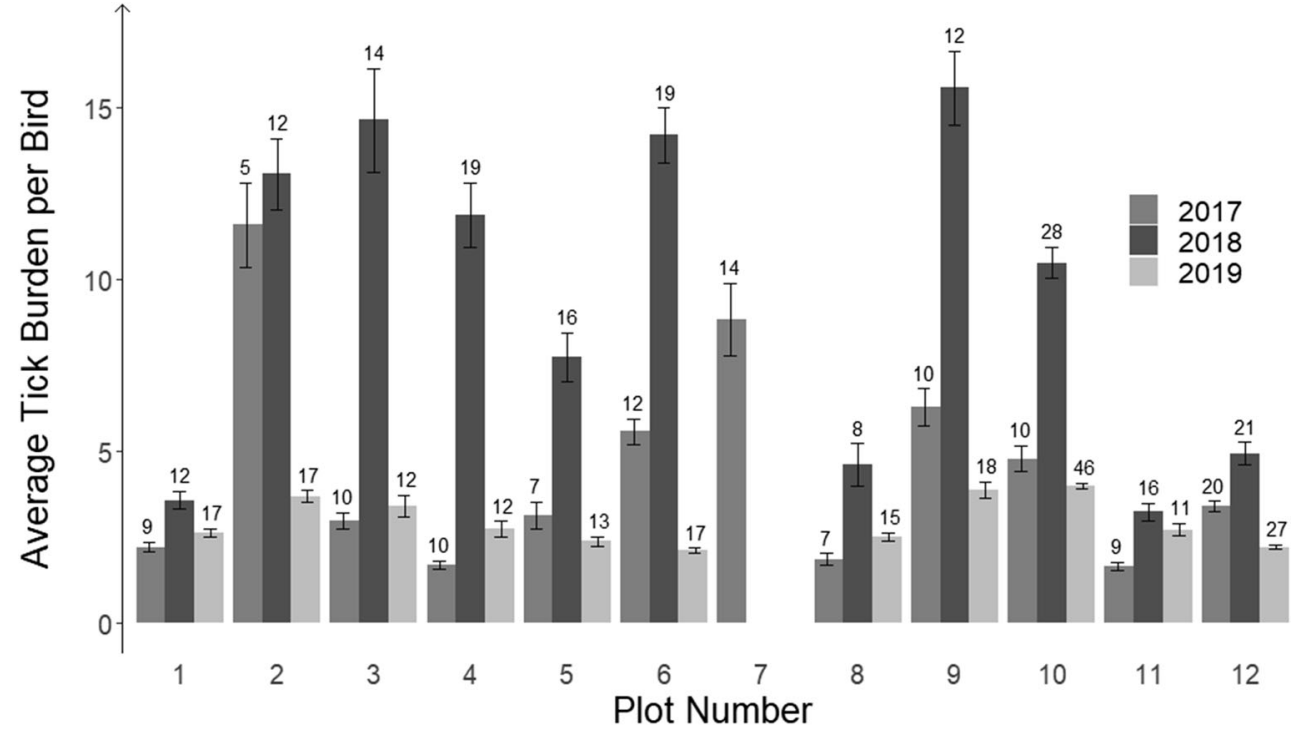

Importantly, body condition and exploration behaviour are (sometimes) correlated (Dosmann et al. 2015; Moiron et al. 2019) but effects of exploration behaviour and body condition on tick burden have not been disentangled. Previously, no relationship had been found between tick infestation and body condition in wild great tits (Heylen and Matthysen 2008; Heylen et al. 2013). Our study only partially confirms this result as body condition did not influence infestation probability, but tick burden of infested birds was negatively correlated to body condition. This negative relationship between body condition and tick burden has been observed in other species, and has been attributed to health reductions due to blood loss (Norte et al. 2013). However, the direction of the effect remains unclear, perhaps because multiple factors are involved. High tick burdens could in fact lower body condition through blood loss (Norte et al. 2013) but, additionally, birds with lower body condition could be more susceptible to tick infestation, and consequently, have higher tick burdens (Norte et al. 2010, 2013). More research is therefore needed to disentangle the pathways by which these patterns are generated.

Individuals were not repeatable in infestation probability, but tick burden was repeatable within infested individuals. This means that phenotypic traits other than those measured here (i.e. exploratory behaviour and body condition) explain tick burdens of infested birds. For instance, grooming behaviour has been shown to shape tick burdens in mammalian species (Keesing et al. 2009) and appears to influence tick infestation patterns in great tits (Fracasso et al. 2019). Additional behaviours such as aggression or foraging
Fig. 3 Infestation probability in relation to exploration score. For illustrational purposes only, the prepared raw data of infestation probability and exploration score were split into 10 quantiles and were averaged to help visualise this relationship. Each point refers to the mean infestation probability and exploration score for the given quantile. Error bars represent the standard error for the respective mean infestation probability. The numbers report the number of records in the respective quantile. The line reports the linear relationship presented in Table 1

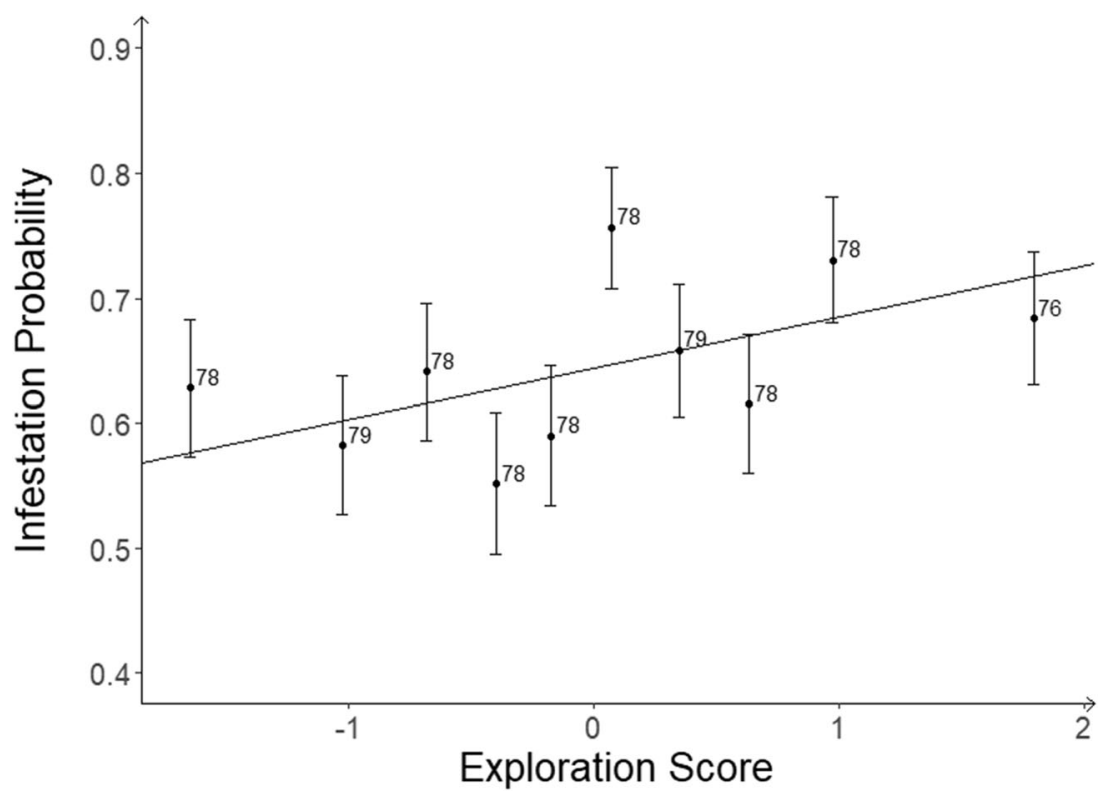


Fig. 4 Tick burden of infested birds in relation to their scaled mass index (i.e. body condition). The line reports the linear relationship presented in Table 1

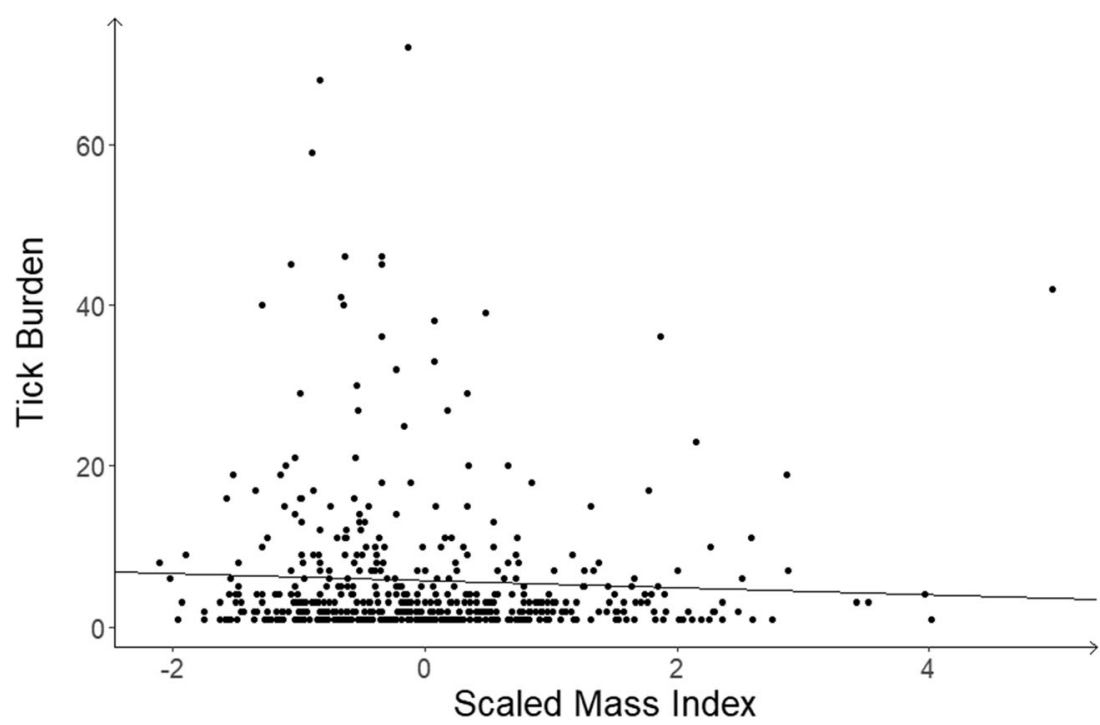

behaviour (see above) may shape tick infestation (Newman et al. 2015; Loss et al. 2016; Kocianová et al. 2017; Zohdy et al. 2017). Our inability to demonstrate that infestation probability was repeatable may also stem a lack of statistical power, a common problem in repeatability analyses of binary traits.

Spatial factors, including nest location, influenced tick infestation. Great tits might choose their breeding site based on several ecological factors, such as resource availability, conspecific density (Spiegel et al. 2015, 2017; Sih et al. 2018), or human disturbance (Hutfluss and Dingemanse 2019). As ticks are heterogeneously distributed over the breeding habitat (Hillyard 1996; Perez et al. 2016), factors influencing great tit habitat choice may also affect tick infestation. Tick abundance is normally affected by micro-spatial differences in, for example, relative humidity (Lindgren et al. 2000; Oorebeek and Kleindorfer 2008; Kiffner et al. 2011), vegetation type or structure (Kiffner et al. 2011; Tack et al. 2012a, b, 2013), or host availability (i.e. rodents, roe deer, etc.) (Oorebeek and Kleindorfer 2008; Kiffner et al. 2011; Takumi et al. 2019). Importantly, our birds were captured approximately over a 1month period (range: 25-29 days), mostly in May each year, which corresponds to the peak of tick abundance (Kurtenbach et al. 2006). Seasonality, such as in weather conditions (e.g. relative humidity), can influence overall tick abundance (Mejlon and Jaenson 1997; Perez et al. 2016) and thus tick burden (Heylen et al. 2013). High host (bird) population density might push some individuals to occupy habitats more suitable for ticks, e.g. more humid habitats with denser vegetation (Tack et al. 2012a, b), and thereby influence tick infestation. This was not the case here as tick infestation did not vary with breeding density. The absence of this effect could be due to confounding effects at different levels of biological variation (van de Pol and Wright 2009). We therefore split
Fig. 5 Infestation probability in relation to recreation pressure. For graphical purposes only, the prepared raw data of infestation probability and recreation pressure were split into 10 quantiles and were averaged to help visualise this relationship. Each point refers to the mean infestation probability and recreational pressure index for the given quantile. Error bars report the standard error for the respective mean infestation probability. The numbers report the number of records in the respective quantile. The line reports the linear relationship presented in Table 1

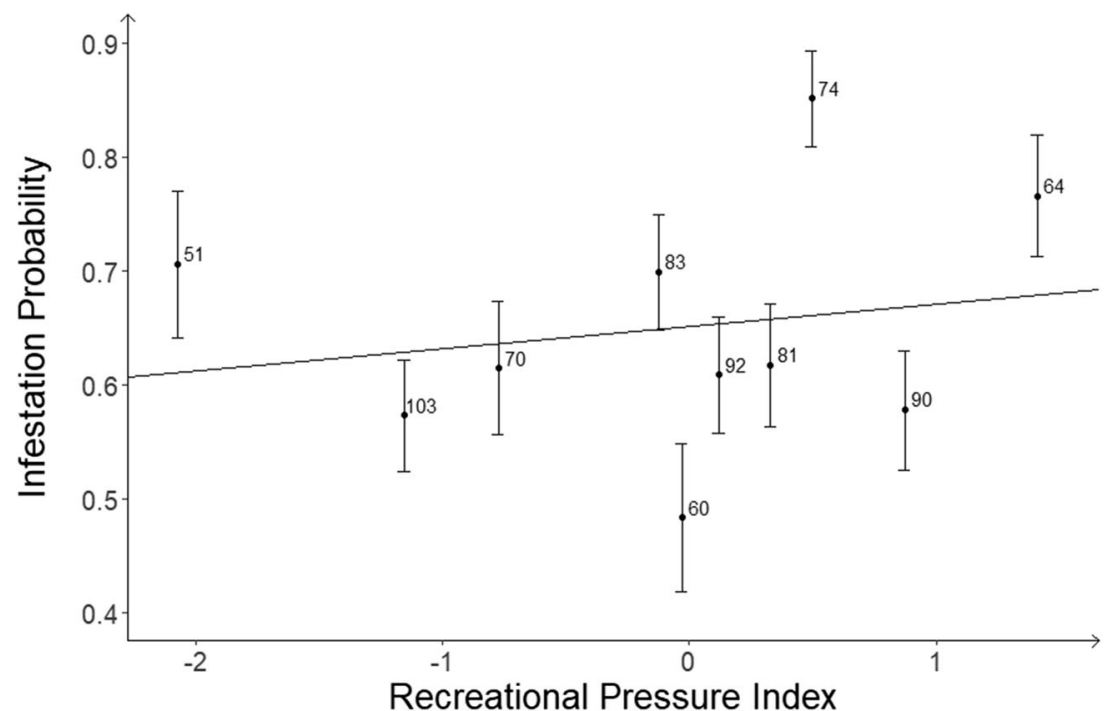


variation in great tit breeding density into temporal and spatial components and tested if this mattered, which was not the case (Supplemental Text 1). As great tits are site faithful (Harvey et al. 1979) and nest box identity predicts tick infestation, future work should consider why individuals nest or potentially do not nest in habitats with high tick abundances. For this, fine-scale data on questing tick densities near occupied nest boxes, and information on ecological variables (i.e. vegetation type, humidity, other host species), would be required. Furthermore, as the average tick burden for any given host will be modified based on the ratio of competent hosts and tick abundance in a given geographic area (Randolph 1998, 2008; Brunner and Ostfeld 2008), the presence of other suitable vertebrate hosts (i.e. rodents and roe deer Capreolus capreolus) may have influenced tick infestation for any particular host species. Testing for interacting effects of multiple host species on tick infestation in great tits may therefore represent a promising avenue for future research.

In conclusion, we showed covariance between exploration behaviour of great tits and their tick infestation probability, with faster explorers having increased infestation probabilities. For the subpopulation of infested birds, however, fast explorers did not carry a larger tick burden among infested birds. Our study thereby supports previous research demonstrating effects of animal personality on susceptibility to tick parasitism (Sih et al. 2018). Tick burden was not influenced by sex or age, but negatively correlated with body condition. Infested individuals were, furthermore, repeatable in tick burden, implying that phenotypic traits other than those considered here explain repeatable differences in tick burden of infested individuals. Finally, recreation pressure tended to predict infestation probability. Our results highlight the complex nature of the parasitic relationship between birds and ticks and the important moderating effect of phenotypic traits specific to individual hosts.

Supplementary Information The online version contains supplementary material available at https://doi.org/10.1007/s00265-021-02972-y.

Acknowledgements We would like to thank all past members of the 'Evolutionary Ecology of Variation' group of the Max Plank Institute for Ornithology, the 'Behavioural Ecology' and 'Evolutionary Biology' groups at the LMU, and Alexander Hutfluss, Sabrina Hepner, Janna Wuelbern, all lab technicians and colleagues at the 'National Reference Centre for Borrelia,' field assistants, and students for help in data collection. Additionally, we would like to thank the reviewers for their comments in preparing this study for publication.

Authors' contributions All co-authors helped in developing the study design. RER and AM collected data, AM managed the database, and RER performed all data analysis with input from NJD and NSB. LCD handled all tick morphological identification. NJD, NSB, VF, and GM provided expert input and guidance for proper model parameters, including development of the screening protocol. RER wrote the manuscript with input and final approval from all co-authors.
Funding Open Access funding enabled and organized by Projekt DEAL. NJD and AM were supported by the German Science Foundation (Grant No. DI 1694/1-1) and the Max Planck Society. NSB was supported by a BioNa - Nachwuchsforscherpreis of the faculty of Biology of the Ludwig Maximilian Universität München.

Data availability The datasets analysed and R-scripts used during the current study are available through the Dryad Digital repository at https://doi.org/10.5061/dryad.v41ns1rtz.

\section{Compliance with ethical standards}

Conflict of interest The authors declare that they have no conflict of interest.

Ethics approval All ethical approval and ethics committee permissions for animals used in this study were granted by the District Government of Upper Bavaria (Regierung von Oberbayern) for Animal Care (Permit Number: ROB-55.2-2532.Vet 02-17-215) in accordance with the $\mathrm{ASAB} / \mathrm{ABS}$ Guidelines for the use of animals in research.

Open Access This article is licensed under a Creative Commons Attribution 4.0 International License, which permits use, sharing, adaptation, distribution and reproduction in any medium or format, as long as you give appropriate credit to the original author(s) and the source, provide a link to the Creative Commons licence, and indicate if changes were made. The images or other third party material in this article are included in the article's Creative Commons licence, unless indicated otherwise in a credit line to the material. If material is not included in the article's Creative Commons licence and your intended use is not permitted by statutory regulation or exceeds the permitted use, you will need to obtain permission directly from the copyright holder. To view a copy of this licence, visit http://creativecommons.org/licenses/by/4.0/.

\section{References}

Abbey-Lee RN, Dingemanse NJ (2019) Adaptive individual variation in phenological responses to perceived predation levels. Nat Commun 10:1601

Abbey-Lee RN, Mathot KJ, Dingemanse NJ (2016) Behavioral and morphological responses to perceived predation risk: a field experiment in passerines. Behav Ecol 27:857-864

Akimov IA, Nebogatkin IV (2016) Ixodid ticks (Acari, Ixodidae) in urban landscapes. A review. Vestn Zool 50:155-162

Aplin LM, Farine DR, Mann RP, Sheldon BC (2014) Individual-level personality influences social foraging and collective behaviour in wild birds. Proc R Soc B 281:20141016

Araya-Ajoy YG, Dingemanse NJ (2017) Repeatability, heritability, and age-dependence of seasonal plasticity in aggressiveness in a wild passerine bird. J Anim Ecol 86:227-238

Araya-Ajoy YG, Kuhn S, Mathot KJ, Mouchet A, Mutzel A, Nicolaus M, Wijmenga JJ, Kempenaers B, Dingemanse NJ (2016) Sources of (co)variation in alternative siring routes available to male great tits (Parus major). Evolution 70:2308-2321

Barber I, Dingemanse NJ (2010) Parasitism and the evolutionary ecology of animal personality. Philos Trans R Soc B 365:4077-4088

Bates D, Maechler M, Bolker B, Walker S (2015) Fitting linear mixedeffects models using lme4. J Stat Softw 67:1-48

Brunner JL, Ostfeld RS (2008) Multiple causes of variable tick burdens on small-mammal hosts. Ecology 89:2259-2272 
Dingemanse NJ, Bouwman KM, van de Pol M, van Overveld T, Patrick SC, Matthysen E, Quinn JL (2012) Variation in personality and behavioural plasticity across four populations of the great tit Parus major. J Anim Ecol 81:116-126

Dingemanse NJ, Moiron M, Araya-Ajoy YG (2020) Individual variation in age-dependent reproduction: fast explorers live fast but senesce young? J Anim Ecol 89:601-613

Dosmann AJ, Brooks KC, Mateo JM (2015) Within-individual correlations reveal link between a behavioral syndrome, condition and cortisol in free-ranging Belding's ground squirrels. Ethology 121: $125-134$

Estrada-Peña A, Nava S, Petney T (2014) Description of all the stages of Ixodes inopinatus n. sp. (Acari: Ixodidae). Ticks Tick Borne Dis 5: 734-743

Evans KL, Gaston KJ, Sharp SP, McGowan A, Simeoni M, Hatchwell BJ (2009) Effects of urbanisation on disease prevalence and age structure in blackbird Turdus merula populations. Oikos 118:774-782

Filippova NA (1977) Arachnida class: ixodid ticks of the subfamily Ixodinae. Fauna SSSR Paukoobraznye, Nauka, Liningrad

Fracasso G, Matthysen E, Dhondt AA, Heylen D (2019) Experimental study of micro-habitat selection by ixodid ticks feeding on avian hosts. Int J Parasitol 49:1005-1014

Gelman A, Su Y-S (2016) arm: data analysis using regression and multilevel/hierarchical models, https://CRAN.R-project.org/ package $=$ arm

Hadfield JD, Wilson AJ, Garant D, Sheldon BC, Kruuk LEB (2010) The misuse of BLUP in ecology and evolution. Am Nat 175:116-125

Hamer SA, Lehrer E, Magle SB (2012) Wild birds as sentinels for multiple zoonotic pathogens along an urban to rural gradient in Greater Chicago, Illinois. Zoonoses Public Health 59:355-364

Harvey PH, Greenwood PJ, Perrins CM (1979) Breeding area fidelity of great tits (Parus major). J Anim Ecol 48:305-313

Heylen D, Adriaensen F, Van Dongen S, Sprong H, Matthysen E (2013) Ecological factors that determine Ixodes ricinus tick burdens in the great tit (Parus major), an avian reservoir of Borrelia burgdorferi s.1. Int J Parasitol 43:603-611

Heylen D, Lasters R, Adriaensen F, Fonville M, Sprong H, Matthysen E (2019) Ticks and tick-borne diseases in the city: role of landscape connectivity and green space characteristics in a metropolitan area. Sci Total Environ 670:941-949

Heylen D, Matthysen E (2008) Effect of tick parasitism on the health status of a passerine bird. Funct Ecol 22:1099-1107

Hillyard PD (1996) Ticks of north-west Europe. Field Studies Council, Schrewsbury, London

Hoodless AN, Kurtenbach K, Nuttall PA, Randolph SE (2003) Effects of tick Ixodes ricinus infestation on pheasant Phasianus colchicus breeding success and survival. Wildl Biol 9:171-178

Houslay TM, Wilson AJ (2017) Avoiding the misuse of BLUP in behavioural ecology. Behav Ecol 28:948-952

Hutfluss A, Dingemanse NJ (2019) Human recreation reduces clutch size in great tits Parus major regardless of risk-taking personality. Behav Ecol 30:1751-1760

Keesing F, Brunner J, Duerr S, Killilea M, LoGiudice K, Schmidt K, Vuong H, Ostfeld RS (2009) Hosts as ecological traps for the vector of Lyme disease. Proc R Soc Lond B 276:3911-3919

Kelly CD (2006) Replicating empirical research in behavioral ecology: how and why it should be done but rarely ever is. Q Rev Biol 81: 221-236

Kiffner C, Stanko M, Morand S, Khokhlova IS, Shenbrot GI, Laudisoit A, Leirs H, Hawlena H, Krasnov BR (2013) Sex-biased parasitism is not universal: evidence from rodent-flea associations from three biomes. Oecologia 173:1009-1022

Kiffner C, Vor T, Hagedorn P, Niedrig M, Rühe F (2011) Factors affecting patterns of tick parasitism on forest rodents in tick-borne encephalitis risk areas, Germany. Parasitol Res 108:323-335
Kocianová E, Rus V, Harû D, Spitalská E (2017) Seasonal infestation of birds with immature stages of Ixodes ricinus and Ixodes arboricola. Ticks Tick Borne Dis 8:423-431

Kurtenbach K, Hanincová K, Tsao JI, Margos G, Fish D, Ogden NH (2006) Fundamental processes in the evolutionary ecology of Lyme borreliosis. Nat Rev Microbiol 4:660-669

Lindgren E, Tälleklint L, Polfeldt T (2000) Impact of climatic change on the northern latitude limit and population density of the diseasetransmitting European tick Ixodes ricinus. Environ Health Perspect 108:119-123

Loss SR, Noden BH, Hamer GL, Hamer SA (2016) A quantitative synthesis of the role of birds in carrying ticks and tick-borne pathogens in North America. Oecologia 182:947-959

Lydecker HW, Banks PB, Hochuli DF (2019) Counting ticks (Acari: Ixodida) on hosts is complex: a review and comparison of methods. J Med Entomol 56:1527-1533

Mejlon HA, Jaenson TGT (1997) Questing behaviour of Ixodes ricinus ticks (Acari: Ixodidae). Exp Appl Acarol 21:747-754

Moiron M, Araya-Ajoy YG, Mathot KJ, Mouchet A, Dingemanse NJ (2019) Functional relations between body mass and risk-taking behavior in wild great tits. Behav Ecol 30:617-623

Moiron M, Mathot KJ, Dingemanse NJ (2018) To eat and not be eaten: diurnal mass gain and foraging strategies in wintering great tits. Proc R Soc B 285:20172868

Moyers SC, Adelman JS, Farine DR, Moore IT, Hawley DM (2018) Exploratory behavior is linked to stress physiology and social network centrality in free-living house finches (Haemorhous mexicanus). Horm Behav 102:105-113

Mysterud A, Byrkjeland R, Qviller L, Viljugrein H (2015) The generalist tick Ixodes ricinus and the specialist tick Ixodes trianguliceps on shrews and rodents in a northern forest ecosystem- a role of body size even among small hosts. Parasit Vectors 8:639

Nakagawa S, Parker TH (2015) Replicating research in ecology and evolution: Feasibility, incentives, and the cost-benefit conundrum. BMC Biol 13:88

Nakagawa S, Schielzeth H (2010) Repeatability for Gaussian and nonGaussian data: a practical guide for biologists. Biol Rev 85:935-956

Newman EA, Eisen L, Eisen RJ, Fedorova N, Hasty JM, Vaughn C, Lane RS (2015) Borrelia burgdorferi sensu lato spirochetes in wild birds in northwestern California: associations with ecological factors, bird behavior and tick infestation. PLoS One 10:e118146

Norte AC, Lobato DNC, Braga EM, Antonini Y, Lacorte G, Gonçalves M, Lopes De Carvalho I, Gern L, Núncio MS, Ramos JA (2013) Do ticks and Borrelia burgdorferi s.l. constitute a burden to birds? Parasitol Res 112:1903-1912

Norte AC, Ramos JA, Sampaio HL, Sousa JP, Sheldon BC (2010) Physiological condition and breeding performance of the Great tit. Condor 112:79-86

Oorebeek M, Kleindorfer S (2008) Climate or host availability: what determines the seasonal abundance of ticks? Parasitol Res 103: 871-875

Ots I, Horak P (1996) Great tits Parus major trade health for reproduction. Proc R Soc Lond B 263:1443-1447

Peig J, Green AJ (2009) New perspectives for estimating body condition from mass/length data: the scaled mass index as an alternative method. Oikos 118:1883-1891

Perez G, Bastian S, Agoulon A, Bouju A, Durand A, Faille F, Lebert I, Rantier Y, Plantard O, Butet A (2016) Effect of landscape features on the relationship between Ixodes ricinus ticks and their small mammal hosts. Parasit Vectors 9:20

Core Team R (2019) R: A language and environment for statistical computing. R Foundation for Statistical Computing, Vienna, Austria http://www.R-project.org

Randolph SE (1998) Ticks are not insects: consequences of contrasting vector biology for transmission potential. Parasitol Today 14:186192 
Randolph SE (2004) Tick ecology: processes and patterns behind the epidemiological risk posed by ixodid ticks as vectors. Parasitology 129:S37-S65

Randolph SE (2008) The impact of tick ecology on pathogen transmission dynamics. In: Ticks: biology, disease and control. Cambridge University Press, Cambridge, pp 40-72

Santicchia F, Romeo C, Ferrari N, Matthysen E, Vanlauwe L, Wauters LA, Martinoli A (2019) The price of being bold? Relationship between personality and endoparasitic infection in a tree squirrel. Mamm Biol 97:1-8

Sih A, Spiegel O, Godfrey S, Leu S, Bull CM (2018) Integrating social networks, animal personalities, movement ecology and parasites: a framework with examples from a lizard. Anim Behav 136:195-205

Snijders L, van Rooij EP, Burt JM, Hinde CA, van Oers K, Naguib M (2014) Social networking in territorial great tits: slow explorers have the least central social network positions. Anim Behav 98:95-102

Spiegel O, Leu ST, Bull CM, Sih A (2017) What's your move? Movement as a link between personality and spatial dynamics in animal populations. Ecol Lett 20:3-18

Spiegel O, Leu ST, Sih A, Godfrey SS, Bull CM (2015) When the going gets tough: behavioural type-dependent space use in the sleepy lizard changes as the season dries. Proc R Soc B Biol Sci 282: 20151768

Stuber EF, Araya-Ajoy YG, Mathot KJ, Mutzel A, Nicolaus M, Wijmenga JJ, Mueller JC, Dingemanse NJ (2013) Slow explorers take less risk: a problem of sampling bias in ecological studies. Behav Ecol 24:1092-1098

Tack W, Madder M, Baeten L, Vanhellemont M, Gruwez R, Verheyen K (2012a) Local habitat and landscape affect Ixodes ricinus tick abundances in forests on poor, sandy soils. Forest Ecol Manag 265:30-36
Tack W, Madder M, Baeten L, Vanhellemont M, Verheyen K (2013) Shrub clearing adversely affects the abundance of Ixodes ricinus ticks. Exp Appl Acarol 60:411-420

Tack K, Madder M, Baeten L, Frenne PDE, Verheyen K (2012b) The abundance of Ixodes ricinus ticks depends on tree species composition and shrub cover. Parasitology 139:1273-1281

Taggart P, Leu S, Spiegel O, Sih A (2018) Endure your parasites: sleepy lizard movement is not affected by their parasites. Can J Zool 96: 1309-1316

Takumi K, Sprong H, Hofmeester TR (2019) Impact of vertebrate communities on Ixodes ricinus-borne disease risk in forest areas. Parasit Vectors 12:434

Titcomb G, Allan BF, Ainsworth T, Henson L, Hedlund T, Pringle RM, Palmer TM, Njoroge L, Campana MG, Fleischer RC, Mantas JN, Young HS (2017) Interacting effects of wildlife loss and climate on ticks and tick-borne disease. Proc R Soc B 284:20170475

van de Pol M, Wright J (2009) A simple method for distinguishing within- versus between-subject effects using mixed models. Anim Behav 77:753-758

Verbeek ME, Drent PJ, Wiepkema PR (1994) Consistent individual differences in early exploratory behaviour of male great tits. Anim Behav 48:1113-1121

Zohdy S, Bisanzio D, Tecot S, Wright PC, Jernvall J (2017) Aggression and hormones are associated with heterogeneity in parasitism and parasite dynamics in the brown mouse lemur. Anim Behav 132: 109-119

Publisher's note Springer Nature remains neutral with regard to jurisdictional claims in published maps and institutional affiliations. 